\title{
Kerr and Raman beam cleanup with supercontinuum generation in multimode microstructure fiber
}

\author{
R. Dupiol ${ }^{1,2}$, K. Krupa ${ }^{3}$, A. Tonello ${ }^{1}$, M. Fabert ${ }^{1}$, D. Modotto $^{3}$, S. Wabnitz ${ }^{2,3}$, G. Millot ${ }^{2}$, and V. Couderc ${ }^{1}$ \\ 1. Université de Limoges, XLIM, UMR CNRS 7252, 123 Avenue A. Thomas, 87060 Limoges, France \\ 2. Université Bourgogne Franche-Comté, ICB UMR CNRS 6303, 9 Avenue A. Savary, 21078 Dijon, France \\ 3. Dipartimento di Ingegneria dell'Informazione, Università di Brescia and INO-CNR, Via Branze 38, 25123 Brescia, Italy \\ richard.dupiol@unilim.fr
}

\begin{abstract}
We experimentally study the interplay of Kerr and Raman beam cleanup in multimode air-silica microstructure optical fiber. The interplay of modal four-wave mixing and Raman scattering leads to high-brightness multimode supercontinuum.(C) 2018 The Author(s)

OCIS codes: (190.4370) Nonlinear optics, fibers; (190.3270) Kerr effect; (190.4380) Nonlinear optics, four-wave mixing; (190.5650) Raman effect; (190.5940) Self-action effects
\end{abstract}

Stimulated Raman scattering (SRS) in graded index (GRIN) multimode fibers (MMFs) permits to convert a multimode, speckled pump into a high brightness, nearly single mode Stokes beam [1,2]. Recent experiments have revealed that GRIN MMFs may also be used for the delivery of high brightness laser beams, based on the mechanism of Kerr spatial beam self-cleaning [3-5]. For beam powers above the threshold for Kerr beam cleaning, frequency conversion of nearly single mode beams across the entire transparency range of silica glass is achieved by the mechanisms of parametric sideband series and supercontinuum generation $[3,4,6,7]$.

In this work, we present what is, to our knowledge, the first observation of Raman beam cleanup, Kerr beam self-cleaning, and supercontinuum generation in a microstructure MMF. We used a specially conceived air-silica microstructure MMF, whose transverse structure strongly differs from that of a standard, weakly guiding GRIN MMF. We demonstrate that, unlike beam cleaning in GRIN fibers, the quality of the pump beam at the output of our microstructure fiber does not monotonically increase with growing input power. The Kerr-induced spatial beam cleaning competes with Raman cleanup. Above a certain threshold power, SRS induced Stokes beam cleaning spoils pump self-cleaning, while still enhancing beam brightness on the infrared side of the supercontinuum spectrum.

Fig.1 shows our microstructure MMF, with hexagonal pure silica core surrounded by three layers of air holes as optical cladding, whose diameters gradually increase with the layer order, so that the effective index profile mimicks that of a GRIN MMF.
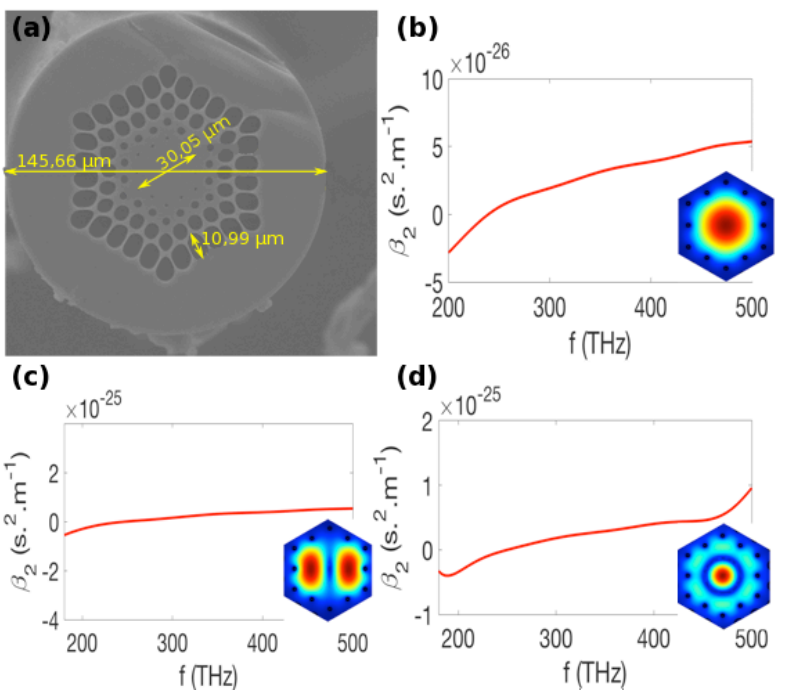

(d)

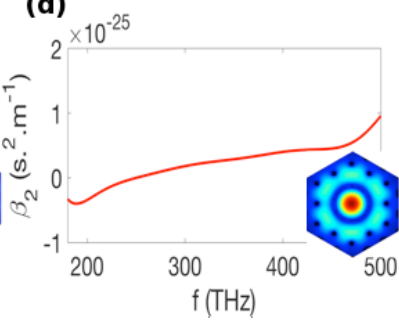

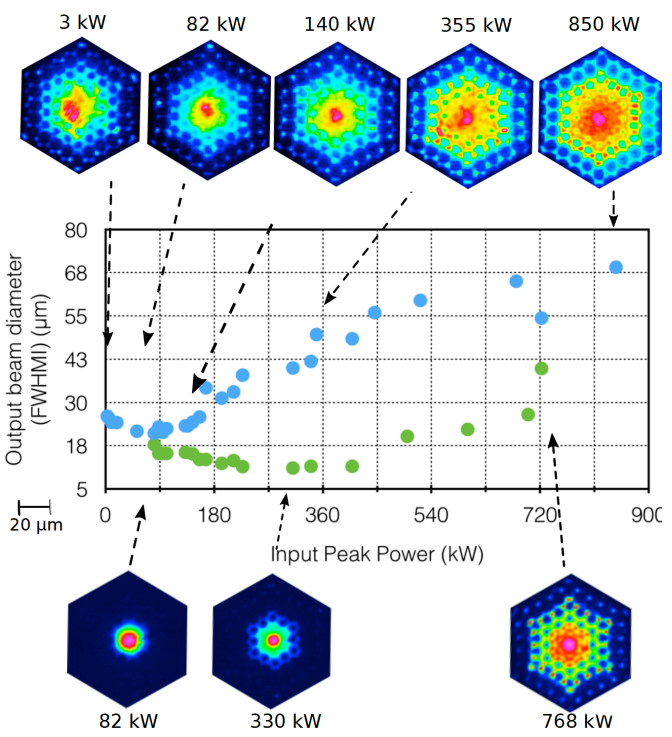

Fig. 1. (a) Cross section image of the microstructure MMF from a scanning electron microscope; numerically calculated intensity of the first three guided modes and their dispersion curves (mode 1 (b), mode 2 (c) and mode 3 (d)) computed at 1064nm; Right panel: power evolution of beam diameter for the pump (blue dots) and the first Raman Stokes (green dots); insets: corresponding output beam patterns for different input peak powers for a 11-m long fiber. 
In our experiments, we used a spatially single-mode laser source at $1064 \mathrm{~nm}$, delivering pulses of $60 \mathrm{ps}$ with the repetition rate of $20 \mathrm{kHz}$. We first studied the power dependence of the output beam shape at both the pump wavelength and at the first Raman Stokes. The right panel in Figure 1 summarizes the power evolution of the transverse beam diameter, measured at the output of 11-m long fiber for both the residual pump and the first Raman Stokes beam. At relatively low input pump powers, the output pump exhibits a typical speckled multimodal structure, with a beam diameter close to the size of the inner silica core When increasing the input pump power above $90 \mathrm{~kW}$, Kerr self-cleaning first leads to a narrowing of the output beam diameter, and then its width starts to grow with power, owing to SRS, which introduces a strong energy depletion in the central part of the beam. Whereas, Figure 2 shows that the Stokes beam diameter shrinks down to about 10 microns in diameter, owing to SRS cleanup.

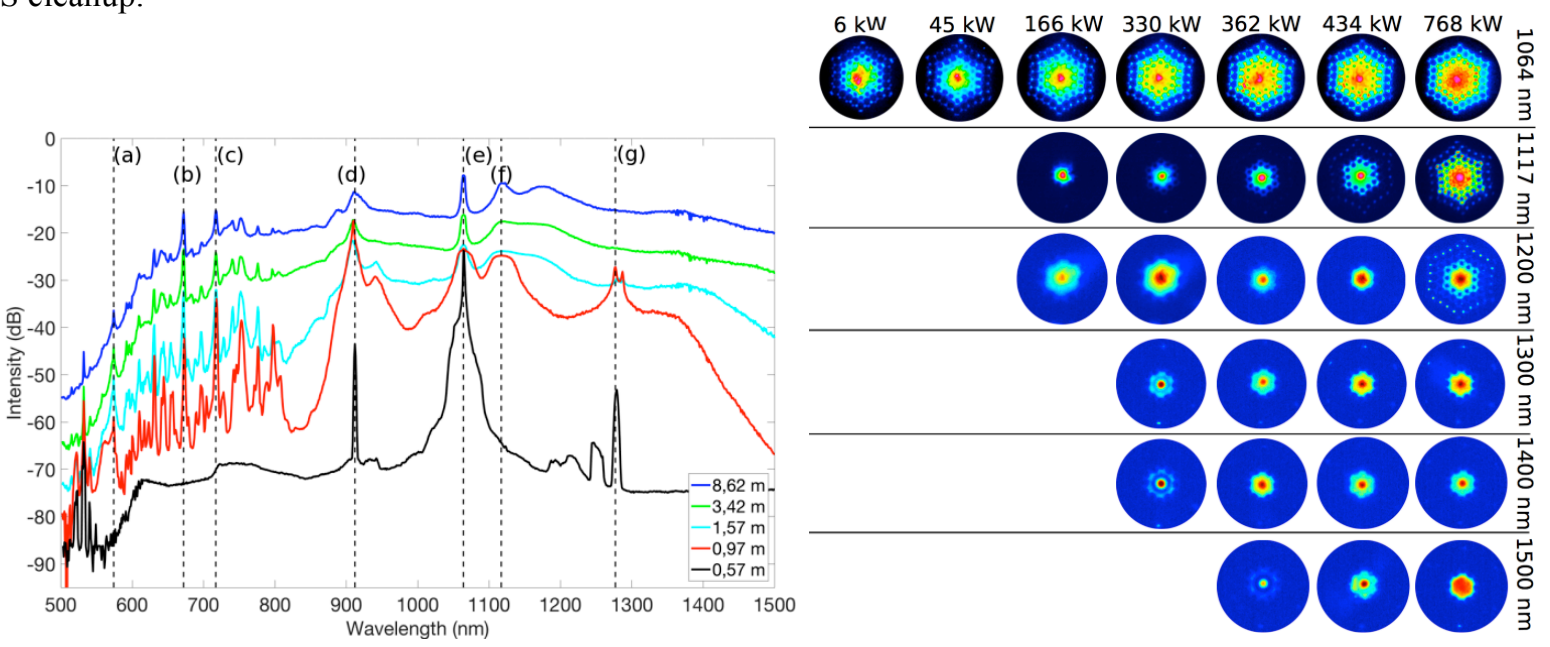

Fig. 2. Output spectrum for different fiber lengths (input peak power: $541 \mathrm{~kW}$ ). Dashed lines correspond to the following wavelengths (detuning from the pump: (a) $574 \mathrm{~nm}(240.5 \mathrm{THz})$, (b) $672 \mathrm{~nm}(164.4 \mathrm{THz})$, (c) $717 \mathrm{~nm}(136.4 \mathrm{THz})$, (d) $912 \mathrm{~nm}$ (46.96 THz), (e) $1064 \mathrm{~nm}$, (f) $1117 \mathrm{~nm}(13.4 \mathrm{THz}),(\mathrm{g}) 1277 \mathrm{~nm}(47.0 \mathrm{THz})$; Right panel: Spatial evolution of the beam profile for different powers and wavelengths (fiber length of $9 \mathrm{~m}$ ).

In Fig.2 we show the evolution of modal four-wave mixing, SRS frequency conversion and supercontinuum generation vs. fiber length: beyond $9 \mathrm{~m}$, a continuum ranging from $500 \mathrm{~nm}$ up to $1800 \mathrm{~nm}$ is generated. In the right panel of Fig. 2 we also present a comprehensive overview of the output beam shape from the 9-m long microstructure MMF, at different wavelengths and for increasing peak powers.

In conclusion, we studied nonlinear beam shaping, frequency conversion and supercontinuum generation in a specialty multimode microstructure fiber. We observed Kerr beam self-cleaning, and its competition with Raman beam cleanup. Raman conversion is particularly efficient in the fiber, which results in a progressive transfer of beam brightness towards the infrared side of the spectrum. This effect is accompanied by a loss of beam quality of the residual pump and the first Stokes wave, once it becomes a pump for higher order Stokes beams.

We acknowledge support from: iXcore research foundation; Labex ACTION program (contract ANR-11-LABX-0001-01); Horiba Medical (Dat@diag); the European Research Council (ERC) under the European Union's Horizon 2020 research and innovation programme, ERC AdG grant agreement No. 740355 and Marie-Sklodowska-Curie grant agreement No. GA-2015713694.

[1] H. Baek, and W. B. Roh, "Single-mode Raman fiber laser based on a multimode fiber", Opt. Lett. 29, 153 (2004).

[2] N. B. Terry, T. G. Alley, and T. H. Russel, "An explanation of SRS beam cleanup in graded-index fibers and the absence of SRS beam cleanup in step-index fibers", Opt. Express. 15, 17509 (2007).

[3] K. Krupa, A. Tonello, A. Barthélémy, V. Couderc, B. M. Shalaby, A. Bendahmane, G. Millot, S. Wabnitz, "Observation of geometric parametric instability induced by the periodic spatial self-imaging on multimode waves", Phys. Rev. Lett. 116, 183901 (2016).

[4] L. G. Wright, Z. Liu, D. A. Nolan, M.-J. Li, D. N. Christodoulides, and F. W. Wise, "Self-organized instability in graded-index multimode fibres", Nat. Photon. 10, 771 (2016).

[5] K. Krupa, A. Tonello, B. M. Shalaby, M. Fabert, A. Barthélémy, G. Millot, S. Wabnitz, and V. Couderc, "Spatial beam self-cleaning in multimode fibres", Nat. Photon. 11, 237 (2017).

[6] G. Lopez-Galmiche, Z. Sanjabi Eznaveh, M. A. Eftekhar, J. Antonio Lopez, L. G. Wright, F. Wise, D. Christodoulides, and R. Amezcua Correa, "Visible supercontinuum generation in a graded index multimode fiber pumped at $1064 \mathrm{~nm}$ ", Opt. Lett. 41, 2553 (2016).

[7] K. Krupa, C. Louot, V. Couderc, M. Fabert, R. Guenard, B. M. Shalaby, A. Tonello, D. Pagnoux, P. Leproux, A. Bendahmane, R. Dupiol, G. Millot, and S. Wabnitz, "Spatiotemporal characterization of supercontinuum extending from the visible to the mid-infrared in a multimode graded-index optical fiber", Opt. Lett. 41, 5785 (2016). 